\title{
VIGENCIA DEL MÉTODO CONTABLE PARA VALORIZAR EL PATRIMONIO DE LAS PYMES
}

\author{
VALIDITY OF THE ACCOUNTING METHOD TO APPRAISE THE ASSETS OF SMES \\ Angel Roberto SAlazar Frisancho* \\ Unidad de Posgrado de la Facultad de Ciencias Contables \\ Universidad Nacional Mayor de San Marcos - UNMSM / Lima - Perú \\ [Recepción: abril de 2016/ Conformidad: mayo 2016]
}

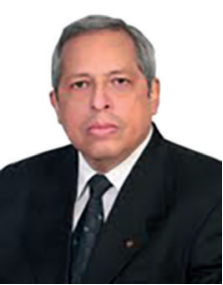

\section{RESUMEN}

El presente artículo expone y sistematiza los fundamentos del método contable para la valorización de las pymes, de un modo analítico y sintético, de acuerdo a sus sus secuencias también se expone la forma de utilizar el método contable para valorizar el patrimonio de las empresas, por parte del profesional contable, teniendo presente los ajustes al valor del patrimonio al aplicar las normas internacionales de contabilidad referidas a las pymes y a los intangibles. El método contable para la valorización del patrimonio de una empresa tiene la característica de movilizar el conjunto de conocimiento de la disciplina contable. Este método pone en acción los principios, normas, procedimientos y técnicas de la xontabilidad con el fin de valorizar el patrimonio de una empresa. El conjunto de conocimientos señalados es el que se imparte en la formación profesional del contador, lo que predispone y prepara a este para la utilización de los conocimientos adquiridos como método de valorización del patrimonio de las empresas.

Finalmente, se llega a la conclusión de que existe una vigencia y reconocimiento del método contable en general para la valorización del patrimonio de las empresas, y por los otros métodos como un un referente.

\section{Palabras Clave:}

Método contable; patrimonio; pymes; activo intangible; valorización del patrimonio.

\begin{abstract}
This paper presents and systematizes the grounds of accounting method for the appraising of SMEs, in an analytical and synthetic way, according to their sequences it is also disclosed the way to use the accounting method to value the assets of the companies -by the accounting professional- taking into consideration the adjustments of the of assets' value in applying the international accounting standards relating to SMEs and the intangibles.

The accounting method for valuing company assets has the feature of mobilizing all knowledge of the accounting discipline. This method puts into action the principles, rules, procedures and accounting techniques in order to appraise the assets of a company. The set of identified knowledge is taught in the professional training of the accountant, which predisposes and prepares this professional for the use of knowledge acquired as a method of valuing the assets of the companies.

Finally, it is concluded that there is a validity and recognition of the general accounting method for valuing the assets of companies, and by other methods as a reference.
\end{abstract}

\section{Keywords:}

Accounting method; assets; small businesses; intangible assets; valuation of assets.

\footnotetext{
* Maestrista en Contabilidad con mención en Banca y Finanzas. Contador Público Colegiado - UNMSM. Email: fris@salazarfrisancho.com
} 


\section{INTRODUCCIÓN}

El problema de la valorización del patrimonio de las empresas en general se plantea y manifiesta en el objetivo del conocimiento del valor (precio) del patrimonio de una empresa. Este objetivo se expresa también, de un modo general, en la interrogante: ¿Cuál es el valor de una empresa? o ¿Cuánto cuesta una empresa?

En el nivel de la teoría y de la ciencia el problema, en general, se plantea a partir del objetivo que es conocer el método y las técnicas que conducen a una correcta valorización del patrimonio de una empresa. De este modo, el objetivo se plantea con la interrogante: ¿Cuál es la teoría y el método adecuado para la valorización correcta del patrimonio de una empresa? ¿Qué metodología científica es la adecuada para la valorización del patrimonio de una empresa?

Así planteado el problema, este nos remite al ámbito de las ciencias y sus técnicas, lo que conduce a conocer la ciencia, que proporciona el método y las técnicas para medir el valor del patrimonio de una empresa. Esto se plantea en la interrogante: ¿Qué ciencia o ciencias, nos permiten conocer el valor del patrimonio de una empresa?

La respuesta, en forma general, tiene que ver con la ciencia económica, la disciplina contable, la ciencia de la administración, y el marketing.

El presente artículo, de modo general, aborda el método apropiado para la valorización de las empresas. Así, busca responder la siguientes pregunta ¿La ciencia contable tiene el método y las técnicas adecuadas para determinar el valor de una empresa?

A partir de esto, se quiere esclarecer los alcances de los procedimientos de la disciplina contable.

\section{MARCO TEÓRICO}

Para el nivel de la contabilidad en su especificidad se expone las definiciones consignadas en la ponencia "Estudio de las normas contables desde una perspectiva lógica y epistemológica y su utilización en los países de América" presentada por el autor conjuntamente con los Contadores Públicos Colegiados Percy Vilchez Olivares y Jesús Capcha Carbajal en XXVII Conferencia Interamericana de Contabilidad 2007:
La contabilidad es una disciplina científica que describe y explica una determinada región de la realidad social, los hechos contables, estas descripciones y explicaciones están constituidos como teoría contable. La contabilidad, también es una disciplina técnico-profesional que prescribe o regula acciones y están constituidos en un sistema normativo: las normas contables(Vilchez et al., 2007).

\section{Teoría Contable}

Jorge Tua Pereda, en su artículo: "La dimensión social de la Contabilidad" señala que "La teoría contable, es un sistema hipotético-deductivo que describe los hechos contables que están presentes en todos los sistemas normativos".

Bajo el enfoque formalizado, la teoría Contable, es un sistema de enunciados preposicionales, para caracterizar los hechos contables, empíricamente contrastables. Por tanto, se enuncian a través de axiomas, postulados, teorías particulares y principios.

\section{Los hechos contables}

La contabilidad habla de hechos contables y constituye todo hecho que tiene un impacto en el patrimonio de la empresa. El gestor de empresas actúa sobre los hechos administrativos, donde un grupo de estos hechos son contables. El contador no actúa sobre los hechos.

Los hechos contables son los sistemas concretos o cosas, también incluye a procesos (producir), sucesos (partes de un proceso),y al estado de cosas (momentos de un proceso). Una vez que el hecho contable ha sido reconocido, se inicia el proceso contable, en el cual entra a operar la norma contable que brinda los criterios de reconocimiento, medición y revelación de los hechos en los estados financieros.

\section{Valorización de empresas}

R. Alonso Sebastian y A. Villa Pérez, en su libro "Valorización de empresas" señalan que:

La valorización de empresas busca la cuantificación en una unidad de medida homogénea (unidades monetarias), de los elementos que forman el patrimonio de la empresa, de su actividad, de su potencialidad y otras característica destacables. El resultado final es la estimación del valor de la empresa. El proceso de valorización obliga a la investigación de las cualidades económicas 
más importantes de los bienes objeto de valoración que condicionan o pueden condicionar su valor (Alonso y Villa, 2007).

Por su parte, Jaensch (1969), considera que, en el caso de las empresas

"la valoración es un sustitutivo del mecanismo del mercado que para ellas como tales no existe y cuyo objeto es acotar los límites del precio, dentro del cual las empresas pueden transaccionarse” (Jaensh, 1969).

\section{EL MÉTODO CONTABLE}

El método contable para la valorización del patrimonio de una empresa tiene la característica de movilizar el conjunto de conocimientos de la disciplina contable. Este método pone en acción los principios, normas, procedimientos y técnicas de la contabilidad con el fin de valorizar el patrimonio de una empresa.

El conjunto de conocimientos, principios, técnicas y procesos utilizados por el método contable para la valorización del patrimonio de una empresa es el que se imparte en la formación profesional de un contador. Esta predispone y prepara al profesional contable para la utilización del método para valorización del patrimonio de las empresas.

Es necesario señalar que si bien existen otros métodos, la contabilidad manifiesta uno inherente a su disciplina. En cambio los otros métodos conciernen a otras disciplinas científicas.

\section{DESARROLLO DEL MÉTODO}

El método contable se sustenta en los elementos que son inherentes a la ciencia de la contabilidad como son el activo, el pasivo, el patrimonio, los ingresos y los gastos.

La esencia de estos elementos se refleja en dos estados financieros: el estado de situación financiera y el estado de resultado integral, que muestran los hechos económicos que suceden dentro de las empresas.

Para que en una operación económica los hechos producidos por una empresa sean considerados elementos de la contabilidad, como activos, pasivos, ingresos, egresos, etc., tiene que cumplirse la condición de que dicha operación económica permita obtener beneficios económicos y que su valor se pueda estimar con fiabilidad.

\section{PROCEDIMIENTO DEL MÉTODO CONTABLE}

El objetivo del método es valorizar la empresa utilizando las políticas contables (principios, métodos, prácticas, técnicas de medición y otros de la contabilidad) y señalando si los valores de los diversos rubros, expuestos por el estado de situación financiera, son valorados racionalmente.

Debemos observar que no es absolutamente exacta la valoración (la contabilidad no es una ciencia exacta) sino dentro de los parámetros en que se desenvuelven los contadores y la actividad empresarial.

Con la utilización del método contable, lo alcanzado en el estado de situación financiera refleja el patrimonio de la entidad.

Por lo expuesto el objetivo del método contable, es determinar si el patrimonio, expuesto en los estados financieros y fundado en el estado de situación financiera presenta el valor real que tiene la empresa. Si satisface al propietario ese valor, y si se encuentra dentro de la racionalidad del mercado, entonces, el método ha cumplido su objetivo: valorizar el patrimonio de una empresa.

En cuanto a las necesidades del método contable, cuando una empresa (cliente) demanda al profesional contable conocer el valor de su patrimonio, los pasos a seguir para este objetivo son los siguientes:

\section{PRIMERO: Identificar si la empresa cotiza en la bolsa de valores o no cotiza}

Cuando una empresa desea valorizar su patrimonio mediante el método contable, se determina la situación en que se encuentra su patrimonio a una fecha determinada, es decir, si se encuentra o no cotizando en un mercado bursátil (bolsa de valores).

Lo indicado se debe a que, conforme el caso, de cotizar o no en una bolsa de valores, el método contable aplicará técnicas distintas de valorización.

\section{SEGUNDO: Si la empresa cotiza en la bolsa de valores}

Cuando la empresa cotiza la bolsa o quiere cotizar en un mercado bursátil, el método contable se toma 
en cuenta el entorno que rodea a la empresa, es decir, la situación económica, la política fiscal, la política tributaria, los convenios bilaterales de estado, la política monetaria, la inflación, la deflación, la situación social, la situación política y otros aspectos.

Las situaciones señaladas inciden en el patrimonio de la empresa que cotiza en bolsa, por ello, el método contable muestra sus efectos en el patrimonio de la misma.

El método contable aplica la técnica de medición denominada cotización bursátil, consistente en mostrar el valor de la cotización en el mercado de la bolsa de valores de las acciones de la empresa. Este valor que se muestra en el patrimonio del estado de situación financiera de la empresa.

\section{TERCERO: Si la empresa no cotiza en bolsa}

El procedimiento para la valorización de las empresas se desarrolla a través de los siguientes pasos:

\section{PASO 1:}

Se identifican cada uno de los rubros de los activos registrados bajo documentos y principios de contabilidad, dándoles su valor histórico.

Los principales rubros son los referidos a cuentas por cobrar, existencias, activos fijos, activos intangibles, inversiones financieras, inversiones inmobiliarias, activos biológicos y otros activos.

PASO 2:

Una vez identificados los rubros a valorizar, según el giro del negocio, se aplican técnicas de medición o valorización en cada uno de los rubros del activo que la empresa mantenga en su estado de situación financiera con el objetivo de compararlo con su valor histórico de forma que muestre la variación en el estado financiero referido y su efecto en el patrimonio de la empresa.

$\mathrm{Al}$ darse este paso pueden ocurrir dos situaciones: el valor histórico se incremento o el valor histórico disminuye, por efecto de la valorización realizada.

Es importante reconocer en este paso, que el producto de las técnicas de medición o valorización aplicadas tiene relación con el concepto de Valor razonable.
A continuación, señalamos las principales técnicas de medición o valorización para cada rubro del activo:

\section{Cuentas por cobrar}

Tasa efectiva

\section{Existencias}

Valor neto de realización

Índice general de precios, en caso de Inflación

Costo de reposición

\section{Activos fijos}

Tasación

Valor presente

Índice general de precios, en caso de inflación

\section{Activos intangibles}

Tasación

Valor presente

Índice general de precios, en caso de inflación

\section{Inversiones financieras}

Método de participación

Valor presente de flujos descontados

Método de consolidación proporcional

Índice general de precios, en caso de inflación

Inversiones inmobiliarias

Método de tasación

Valor presente

Índice general de precios, en caso de inflación

\section{Activos biológicos}

Valor razonable

Costos estándar.

\section{PASO 3:}

Se identifican cada uno de los rubros de los pasivos registrados bajo documentos y principios de contabilidad, dándoles su valor histórico. Los principales rubros son los referidos a cuentas por pagar, obligaciones financieras y provisiones diversas.

PASO 4:

Una vez identificados los rubros a valorizar en base al giro del negocio, se aplica las técnicas de medición o valorización en cada uno de los rubros 
del pasivo que la empresa mantenga en su estado de situación financiera, con el objetivo de compararlo con su valor histórico de forma que muestre la variación en el estado financiero referido y su efecto en el patrimonio de la empresa.

En este caso puede darse dos situaciones, el valor histórico se incremente o el valor histórico disminuya, por efecto de la valorización realizada.

Es importante tener en cuenta en este paso las técnicas de medición o valorización que son aplicadas en relación con el concepto de valor razonable.

A continuación, señalamos las principales técnicas de medición o valorización para cada rubro del pasivo:

\section{Cuentas por pagar}

Método de tasa efectiva

Obligaciones financieras

Método de tasa efectiva

Método de participación

Valor presente

Provisiones

Estudios actuariales.

\section{PASO 5:}

Dados los pasos expuestos, se da el último paso, que viene a ser el de formular el estado de situación financiera en el sistema contable, lo que permitirá determinar el patrimonio de la empresa como resultado de establecer el Activo - Pasivo = Patrimonio. A continuación, se expone lo indicado en el siguiente gráfico:

Figura No 01:

Ecuación contable que determina el patrimonio de una empresa.

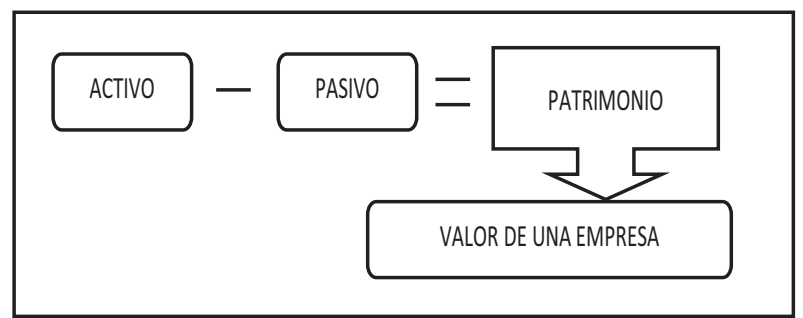

Fuente: Elaboración propia.

\section{OTROS MÉTODOS}

A continuación, se indican algunos métodos denominados "dinámicos" por algunos autores, que se caracterizan por que se toma en cuenta el entorno que rodea a la empresa y los planes de negocios que se formulan.

\section{Valor bursátil}

Es el que resulta de multiplicar el precio de la acción en el mercado de una empresa que cotiza en bolsa, por el número de acciones que mantiene en el capital social.

\section{Flujo de utilidades descontadas}

Se toma la proyección de utilidades de la empresa en unos periodos determinados y se traen a un valor presente neto.

$$
\begin{aligned}
& \qquad P=\sum^{n} \frac{U n}{(1+k)^{n}} \\
& \mathbf{P}=\text { Valor a calcular } \\
& \mathbf{U}=\text { Utilidades proyectadas } \\
& \mathbf{K}=\text { tasa de descuento } \\
& \mathbf{N}=\text { Periodo de tiempo }
\end{aligned}
$$

Es más apropiada su aplicación para empresas que realizan sus operaciones al contado.

\section{Flujos de caja descontados}

Se hace una proyección del flujo de caja por un período determinado y se descuenta o se trae a un valor presente neto.

$$
P=\sum_{(m)}^{n} \frac{F t}{(1+k)^{n}}
$$

$$
\begin{aligned}
& \mathbf{P}=\text { Valor a calcular } \\
& \mathbf{F t}=\text { Flujo neto proyectado } \\
& \mathbf{k}=\text { Tasa de descuento }=\text { Periodo de tiempo }
\end{aligned}
$$

Obedece a la marcada tendencia de las finanzas a utilizar los flujos de cada periodo de tiempo como un método de valuación. 
Tabla No 01:

Ventajas, desventajas y posibilidades del método contable

\begin{tabular}{|c|c|}
\hline VENTAJA & MÉTODO CONTABLE \\
\hline 1. Demanda & $\begin{array}{l}\text { Es el más requerido en el universo empresarial para la valorización del patrimonio de } \\
\text { las empresas. }\end{array}$ \\
\hline 2. Criterio de valor & $\begin{array}{l}\text { Consigna el valor del patrimonio de las empresas en base a documentos consignados } \\
\text { en los libros contables, comprobables y } \\
\text { demostrables. }\end{array}$ \\
\hline 3. Metodología & $\begin{array}{l}\text { Su procedimiento se basa en principios, técnicas, contables que compromete a todo el } \\
\text { sistema de la ciencia contable. }\end{array}$ \\
\hline $\begin{array}{l}\text { 4. Valor referen } \\
\text { otras valorizacic }\end{array}$ & $\begin{array}{l}\text { Es tomado en cuenta por los otros métodos de valorización de las empresas como pun- } \\
\text { to de comparación o aproximación al valor de su patrimonio. }\end{array}$ \\
\hline $\begin{array}{l}\text { 5. Valor referencial para el } \\
\text { mercado }\end{array}$ & $\begin{array}{l}\text { El valor obtenido por el método contable del patrimonio de la empresa es referencial o } \\
\text { base para el mercado en sus movimientos de oferta y demanda. }\end{array}$ \\
\hline DESVENTAJA & MÉTODO CONTABLE \\
\hline 1. Exposición del método & $\begin{array}{l}\text { El método contable a pesar de ser un paradigma contable, para el profesional contable, } \\
\text { no está expuesto, y descrito como corresponde a una tecnología. Asi, no están consigna- } \\
\text { das en un tratado o en un manual especializado los pasos o secuencias a seguir a la hora } \\
\text { de valorizar una empresa. }\end{array}$ \\
\hline 2. Tiempo & $\begin{array}{l}\text { Por su carácter histórico, el método no puede consignar las tendencias futuras que influ- } \\
\text { yen en la valorización del patrimonio de una empresa. }\end{array}$ \\
\hline 3. Criterio & $\begin{array}{l}\text { Por basarse en documentos que están registrados en los libros contables, cuando no } \\
\text { existe una actitud ética del profesional contable puede conllevar a alteraciones de los } \\
\text { valores en la valorización del patrimonio de una empresa. }\end{array}$ \\
\hline 4. Valores no consignados & $\begin{array}{l}\text { El método no consigna la totalidad de los valores contenidos en el patrimonio de una } \\
\text { empresa, principalmente, los llamados intangibles. En el mundo moderno, donde los } \\
\text { intangibles dominan, esta limitación es importante, ya que la contabilidad no consigna } \\
\text { intangibles como el posicionamiento, el prestigio, la confianza, la innovación, el lideraz- } \\
\text { go, entre otros aspectos. }\end{array}$ \\
\hline 5. Competencia & $\begin{array}{l}\text { El método contable está en competencia con otros métodos que tienen la ventaja de } \\
\text { utilizar otros conceptos, principios e instrumentos estadísticos y matemáticos para me- } \\
\text { dir el valor. }\end{array}$ \\
\hline POSIBILIDADES & MÉTODO CONTABLE \\
\hline $\begin{array}{l}\text { Desarrollo de la normativi- } \\
\text { dad contable internacional }\end{array}$ & $\begin{array}{l}\text { Las posibilidades de perfeccionamiento y desarrollo del método contable para su adap- } \\
\text { tación a las nuevas tendencias económicas de la sociedad están dadas por la moderni- } \\
\text { zación de la ciencia contable en la aplicación del valor razonable en los activos de la } \\
\text { empresa, de acuerdo a la NIIF para las pymes y a las NIIF completas. }\end{array}$ \\
\hline
\end{tabular}

Elaboración propia. 


\section{VALOR DE MERCADO}

Se aplica básicamente en empresas que se encuentran en liquidación. El inconveniente que tiene es cuando que los bienes pierden su valor cuando la la empresa se encuentra en esa etapa.

\section{Enfoque de creación de costo}

A veces las compañías o las personas compran una empresa sólo para evitar las dificultades de empezar desde cero. El comprador calcula el precio en base a los costos de su proyecto de organizar personal, obtener licencias, compra de activos fijos y el desarrollo de activos intangibles, como marcas, contratos, etc. Además, le suma una pequeña prima por el esfuerzo y el tiempo que se está ahorrando.

\section{Valor de activos específicos intangibles}

En ocasiones es el único enfoque que se revisa en una venta. Significa que es preferible comprar el activo intangible que crearlo. Una aplicación común de este método es la adquisición de una base de clientes. Esto sucede en industrias como las compañías de seguros, las agencias de publicidad, entre otras.

\section{CONCLUSIONES}

1. El método contable en general se encuentra vigente para la valorización del patrimonio de las pymes, y es reconocido en la aplicación de los otros métodos como un valor referencial

2. Existe la carencia o el vacío de una exposición directa, en un tratado sobre el método contable para la valorización de las pymes.

\section{REFERENCIAS BIBLIOGRÁFICAS}

1. Percy Vilchez Olivares, Jesús Capcha Carbajal, Angel Salazar Frisancho, XXVII Conferencia Interamericana de Contabilidad, 2007, Estudio de las normas contables desde una perspectiva lógica y epistemológica y su utilización en los países de América.

2. ALONSO R. \& VILLA, A., (2007). Valoración de empresas: teoria y casos prácticos. Madrid: Editorial Mundi Prensa, España. 